\title{
THE
}

$5-1982$

\section{Antiferromagnetic Long-Range Order in the Anisotropic Quantum Spin Chain}

Josef Kurmann

Harry Thomas

Gerhard Müller

University of Rhode Island, gmuller@uri.edu

Follow this and additional works at: https://digitalcommons.uri.edu/phys_facpubs

Terms of Use

All rights reserved under copyright.

\section{Citation/Publisher Attribution}

Kurmann, J., Thomas, H., \& Müller, G. (1982). Antiferromagnetic long-range order in the anisotropic quantum spin chain. Physica A: Statistical Mechanics and its Applications, 112(1-2), 235-255. doi: 10.1016/0378-4371(82)90217-5

Available at: http://dx.doi.org/10.1016/0378-4371(82)90217-5

This Article is brought to you for free and open access by the Physics at DigitalCommons@URI. It has been accepted for inclusion in Physics Faculty Publications by an authorized administrator of DigitalCommons@URI. For more information, please contact digitalcommons-group@uri.edu. 


\title{
Antiferromagnetic Long-Range Order in the Anisotropic Quantum Spin Chain
}

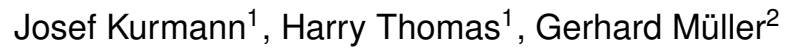 \\ 1 Institut für Physik, Universität Basel, CH-4056 Basel, Switzerland \\ 2 Department of Physics, University of Rhode Island, Kingston RI 02881, USA
}

\begin{abstract}
This is a study of the ground-state properties of the one-dimensional spin-s $(1 / 2<s<\infty)$ anisotropic $X Y Z$ antiferromagnet in a magnetic field of arbitrary direction. It provides the first rigorous results for the general case of this model in non-zero field. By exact calculations we find the existence of an ellipsoidal surface $\mathbf{h}=\mathbf{h}_{N}$ in field space where the ground state is of the classical two-sublattice Néel type with non-zero antiferromagnetic long-range order. At $h_{N}$ there are no correlated quantum fluctuations. We give upper and lower bounds for the critical field $h_{c}$ where antiferromagnetic long-range order is suppressed by the field. The zero-temperature phase diagrams are discussed for a few representative cases.
\end{abstract}

\section{Introduction}

As fundamental results of the theory of critical phenomena it has been found that the importance of fluctuations in a system of interacting spins depends crucially on

(i) the dimensionality of the system,

(ii) the range of interaction,

(iii) the symmetry of the Hamiltonian in spin space,

(iv) at $T \rightarrow 0$, the question whether the spins are treated quantum-mechanically or as classical n-vectors.

Accordingly, among the most strongly fluctuating systems of interacting spins are one-dimensional (1D) spin- $\frac{1}{2}$ chains with nearest-neighbour exchange coupling. Here the thermal fluctuations prevent ordering at any non-zero temperature, and in cases where the Hamiltonian has a continuous rotational symmetry in spin space, the zero-point fluctuations may destroy long-range order (LRO) even at $\mathrm{T}=0$ [1]. In the light of these findings it is of particular interest to study the ground-state (GS) properties of the 1D quantum antiferromagnet (AFM) in a magnetic field. The Hamiltonian reads

$$
\mathcal{H}=\sum_{l=1}^{N}\left\{J_{x} S_{l}^{x} S_{l+1}^{x}+J_{y} S_{l}^{y} S_{l+1}^{y}+J_{z} S_{l}^{z} S_{l+1}^{z}-\mathbf{h} \cdot \mathbf{S}_{l}\right\} .
$$

The exchange parameters $J_{\mu}$ are considered to be non-negative. We shall see that the GS properties depend strongly on the commutability of the exchangeand Zeeman parts $\mathcal{H}_{\mathrm{EX}}$ and $\mathcal{H}_{\mathrm{ZE}}$ of the Hamiltonian. For the $s=\frac{1}{2} \mathrm{XYZ}$ model (1.1) at $h=0$, a number of exact results such as the energies of the GS [2] and of the low-lying excited states [3] are already known. The present work contains the first rigorous results for the general XYZ model in non-zero field. In order to emphasize quantum effects we shall compare our results for the quantum chain (1.1) with those of the classical counterpart (1.1) where the spin operators are replaced by three-dimensional vectors of length $s$.

It is evident from all known results on $T=0$ properties of the 1D AFM (1.1) in zero and nonzero magnetic field that the GS is usually very complicated [4-13]. Below a certain critical value $h_{c}$ of the field, the GS either displays true AFM LRO or only "incipient" LRO, depending on the symmetry of the Hamiltonian. In the latter case the asymptotic behavior $R \rightarrow \infty$ of the correlation functions $\left\langle S_{l}^{\mu} S_{l+R}^{\mu}\right\rangle$ is governed by a power-law decay. In the case of true LRO the appropriate 
correlation function approaches a constant $\left\langle S_{l}^{\mu}\right\rangle^{2}$, and the correlations of the fluctuations $S_{l}^{\mu}-\left\langle S_{l}^{\mu}\right\rangle$ decay exponentially.

In a recent study on the isotropic $s=\frac{1}{2} \mathrm{XY} \operatorname{AFM}\left(J_{x}=J_{y}=J, J_{z}=0\right)$ with an in-plane field $h=h_{x}$ [14] we discovered that at the special value $h_{N}=\sqrt{2} J$ of the field the GS becomes identical to the GS of the corresponding classical chain, i.e. it factorises into single-site states exhibiting the same expectation values $\left\langle S_{l}^{\mu}\right\rangle$ as in the classical two-sublattice Néel-type state with spins of the two sublattices being in a spin-flop configuration within the XY plane. The quantum fluctuations which are considerable both below and above $h_{N}$ become completely uncorrelated at this particular value $h_{N}$. The existence of a quasi-classical GS is actually a general feature of the anisotropic quantum AFM chain in a magnetic field. In section 2 we present a general solution for the conditions under which a Néel-type GS exists for the Hamiltonian (1.1) with arbitrary $s$. We give explicit results for the GS configurations for fields varying in either of the coordinate planes in spin space. In section 3 we elucidate the role of the particular field $h_{N}$ in the classical limit. For this purpose we discuss briefly the effect of a magnetic field on the GS and the spin-wave normal modes of the classical chain. The aim of section 4 is to determine the critical field $h_{c}$ where (true or incipient) AFM LRO is suppressed by the magnetic field. In section 5 we use these results for a discussion of the phase diagrams of a few representative classes of system (1.1).

\section{Néel ground state of the quantum antiferromagnet}

In this section we investigate the existence of a Néel-type GS of Hamiltonian (1.1). It is convenient to treat first the case $s=\frac{1}{2}$, and then generalize the results to arbitrary $s$. In a first step we look for an eigenstate of (1.1) which factorises into alternating single-site states as follows:

$$
\begin{aligned}
& |N\rangle=\prod_{l=1}^{N}\left|\psi_{l}\right\rangle, \\
& \left|\psi_{2 l-1}\right\rangle=a_{1}|2 l-1, \uparrow\rangle+b_{1}|2 l-1, \downarrow\rangle, \\
& \left|\psi_{2 l}\right\rangle=a_{2}|2 l, \uparrow\rangle+b_{2}|2 l, \downarrow\rangle .
\end{aligned}
$$

We use $z$ as quantization axis. It is useful to represent the complex coefficients $a_{i}$ and $b_{j}$ in terms of angular coordinates, $\theta_{j}, \phi_{j}, j=1,2$ :

$$
\begin{array}{ll}
a_{1}=\cos \frac{\theta_{1}}{2} \exp \left(-\imath \frac{\phi_{1}}{2}\right), & b_{1}=\sin \frac{\theta_{1}}{2} \exp \left(\imath \frac{\phi_{1}}{2}\right), \\
a_{2}=\cos \frac{\theta_{2}}{2} \exp \left(-\imath \frac{\phi_{2}}{2}\right), & b_{2}=\sin \frac{\theta_{2}}{2} \exp \left(\imath \frac{\phi_{2}}{2}\right),
\end{array}
$$

The resulting expectation values of the single-site spin components are

$$
\begin{aligned}
& \left\langle\psi_{2 l-1}\left|S_{2 l-1}^{x}\right| \psi_{2 l-1}\right\rangle=\frac{1}{2} \sin \theta_{1} \cos \phi_{1}, \\
& \left\langle\psi_{2 l-1}\left|S_{2 l-1}^{y}\right| \psi_{2 l-1}\right\rangle=\frac{1}{2} \sin \theta_{1} \sin \phi_{1}, \\
& \left\langle\psi_{2 l-1}\left|S_{2 l-1}^{z}\right| \psi_{2 l-1}\right\rangle=\frac{1}{2} \cos \theta_{1},
\end{aligned}
$$

and analogous expressions for sites $2 l$. This is equivalent to saying that the spins of one sublattice $(2 l-1)$ are pointing in direction $\left(\theta_{1}, \phi_{1}\right)$ and those of the other sublattice $(2 l)$ in direction $\left(\theta_{2}, \phi_{2}\right)$ of a polar coordinate system. Thus, for the particular Néel state (2.1), the original nontrivial many-body problem reduces to a simple eigenvalue problem involving only two lattice sites:

$$
\mathcal{H}_{l, l+1}\left|\psi_{l}\right\rangle\left|\psi_{l+1}\right\rangle=\epsilon_{N}\left|\psi_{l}\right\rangle\left|\psi_{l+1}\right\rangle,
$$

with

$$
\mathcal{H}_{l, l+1}=J_{x} S_{l}^{x} S_{l+1}^{x}+J_{y} S_{l}^{y} S_{l+1}^{y}+J_{z} S_{l}^{z} S_{l+1}^{z}-\frac{1}{2}\left[h_{x}\left(S_{l}^{x}+S_{l+1}^{x}\right)+h_{y}\left(S_{l}^{y}+S_{l+1}^{y}\right)+h_{z}\left(S_{l}^{z}+S_{l+1}^{z}\right)\right] .
$$


Apart from the solution of (2.4) for $|\mathbf{h}| \rightarrow \infty$ which is not interesting for our purposes, we find that there exists a solution with energy (per site)

$$
\epsilon_{N}=E_{N} / N=-\frac{1}{4}\left(J_{x}+J_{y}+J_{z}\right)
$$

for finite fields, provided the field vector points to the surface of an ellipsoid characterized by

$$
\frac{h_{x}^{2}}{\left(J_{x}+J_{y}\right)\left(J_{x}+J_{z}\right)}+\frac{h_{y}^{2}}{\left(J_{x}+J_{y}\right)\left(J_{y}+J_{z}\right)}+\frac{h_{z}^{2}}{\left(J_{x}+J_{z}\right)\left(J_{y}+J_{z}\right)}=1 .
$$

In other words, we can freely choose a field direction $(\Theta, P h i)$ in spin space such that $\mathbf{h}=$ $h(\sin \Theta \cos \Phi, \sin \Theta \sin \Phi, \cos \Theta)$, and shall always find a Néel-type eigenstate if the magnitude $h$ of the field takes the value

$$
h_{N}=\left[\frac{\left(J_{x}+J_{y}\right)\left(J_{x}+J_{z}\right)\left(J_{y}+J_{z}\right)}{\left(J_{y}+J_{z}\right) \sin ^{2} \Theta \cos ^{2} \Phi+\left(J_{x}+J_{z}\right) \sin ^{2} \Theta \sin ^{2} \Phi+\left(J_{x}+J_{y}\right) \cos ^{2} \Theta}\right]^{1 / 2} .
$$

We now show that the existence of a Néel eigenstate $|N\rangle$ for $s=\frac{1}{2}$ implies the existence of a Néel eigenstate for any $s$. For this purpose, we note that the unitary transformation $U_{l}$ to a new basis consisting of the state

$$
\left|\psi_{l}\right\rangle=a_{l}|l, \uparrow\rangle+b_{l}|l, \downarrow\rangle
$$

given by $(2.1 \mathrm{~b}, \mathrm{c})$, and an orthogonal state

$$
\left|\varphi_{l}\right\rangle=-b_{l}^{*}|l, \uparrow\rangle+a_{l}^{*}|l, \downarrow\rangle
$$

with $a_{l}, b_{l}$ given by $(2.2)$, defines a rotation $R_{l}$ of the spin vector $\mathbf{S}_{l}$

$$
U_{l}^{-1} S_{l}^{\mu} U_{l}=\sum_{\mu^{\prime}} R_{l}^{\mu \mu^{\prime}} S_{l}^{\mu^{\prime}}
$$

We call the new spin components $S_{l}^{\xi, \eta, \zeta}$. Then the local $\zeta$-axis represents the spin direction at site $l$ in the Néel state. (There exists actually a whole family of such transformations, because $(2.9 \mathrm{~b})$ may still be multiplied by an arbitrary phase factor, corresponding to a rotation about the $\zeta$-axis).

If the Hamiltonian $\mathcal{H}$ has a Néel eigenstate (2.1), then the transformed Hamiltonian

$$
\mathcal{H}^{\prime}=U^{-1} \mathcal{H} U
$$

where $U=\Pi_{l} U_{l}$, has a ferromagnetic eigenstate. Expressing $\mathcal{H}^{\prime}$ in terms of the operators $S_{l}^{\zeta}$ and $S_{l}^{ \pm}=S_{l}^{\xi} \pm \imath S_{l}^{\eta}$,

$$
\begin{aligned}
\mathcal{H}^{\prime}= & \frac{1}{2} \sum_{\mathrm{nn}}\left[A S_{l}^{\zeta} S_{l^{\prime}}^{\zeta}+\left(B S_{l}^{-}+B^{*} S_{l}^{+}\right) S_{l^{\prime}}^{\zeta}+\left(C S_{l}^{-} S_{l^{\prime}}^{-}+C^{*} S_{l}^{+} S_{l^{\prime}}^{+}\right)+D S_{l}^{+} S_{l^{\prime}}^{-}\right] \\
& -\sum_{l}\left[h_{\zeta} S_{l}^{\zeta}+h_{+} S_{l}^{-}+h_{-} S_{l}^{+}\right]
\end{aligned}
$$

where the sum nn is over nearest neighbours, the $A, B, C$ are linear combinations of the exchange constants $J_{\mu}$ and $h_{\zeta}$ and $h_{ \pm}=h_{\xi} \pm \imath h_{\eta}$ represent the components of $\mathbf{h}$ along the new axes, then we find as conditions for a ferromagnetic eigenstate with spin direction along $\zeta$

$$
C=0, \quad B s+h_{+}=0 .
$$

Now, the rotations $R_{l}$ defined by (2.10) may be applied to the spin operators for any spin quantum number $s$. In other words, the $\mathrm{SU}(2)$ unitary transformation $(2.9)$ induces an $\mathrm{SU}(2 s+1)$ unitary transformation by the requirement that both define the same rotation for spin vectors. One can therefore transform the Hamiltonian for arbitrary spin s to the same form (2.12) as above. This 
proves the following theorem: If $\mathcal{H}$ has a Néel eigenstate for exchange constants $J_{\mu}$ and field $\mathbf{h}$ for $s=\frac{1}{2}$, then $\mathcal{H}$ has also a Néel eigenstate for the same exchange constants and field $2 s h$ for arbitrary $s$.

In order to prove that the Néel state $|N\rangle$ is the GS of $\mathcal{H}$, it is sufficient to show that $\epsilon_{N}$ is the GS energy of the pair Hamiltonian (2.5). The proof is especially simple if the field is along one of the axes. For $h=h_{x}$, we find that we can write the pair Hamiltonian in the form

$$
\begin{aligned}
\mathcal{H}_{l, l+1}-\epsilon_{N}= & \frac{1}{2}\left(J_{x}+J_{y}\right)\left[\left(s-S_{l}^{\zeta}\right)\left(s-S_{l+1}^{\zeta^{\prime}}\right)+\left(s-S_{l}^{\zeta^{\prime}}\right)\left(s-S_{l+1}^{\zeta}\right)\right] \\
& +J_{z}\left[s^{2}-\left(S_{l}^{\xi} S_{l+1}^{\xi}+S_{l}^{\eta} S_{l+1}^{\eta}+S_{l}^{\zeta} S_{l+1}^{\zeta}\right)\right]-\left(h-h_{N}^{x}\right) S_{l}^{x},
\end{aligned}
$$

where $S_{l}^{\zeta^{\prime}}$ refers to the spin configuration shifted by a lattice constant, and $\epsilon_{N}=-s^{2}\left(J_{x}+J_{y}+J_{z}\right)$. For $h=h_{N}^{x}$, we have thus expressed $\mathcal{H}_{l, l+1}-\epsilon_{N}$ as a sum of positive operators, which proves that $\left|\psi_{l}\right\rangle\left|\psi_{l+1}\right\rangle$ is the GS of $\mathcal{H}_{l, l+1}$ and therefore $|N\rangle$ is the GS of $\mathcal{H}$. For $s=\frac{1}{2}$, we have checked the GS property of $\left|\psi_{l}\right\rangle\left|\psi_{l+1}\right\rangle$ numerically for various sets of exchange constants and fields in arbitrary directions on the ellipsoid (2.7). But for $s>\frac{1}{2}$ and general field direction we have as yet found no formal proof.

The spin configuration of the Néel state is determined by the eigenvalue equation (2.4) which leads in terms of the new variables

$$
\theta=\frac{1}{2}\left(\theta_{1}+\theta_{2}\right), \quad \phi=\frac{1}{2}\left(\phi_{1}+\phi_{2}\right) ; \quad \alpha=\frac{1}{2}\left(\theta_{1}-\theta_{2}\right), \quad \beta=\frac{1}{2}\left(\phi_{1}-\phi_{2}\right)
$$

to the following equations

$$
\left[\begin{array}{cccccc}
J_{y}+J_{z} & 0 & 0 & 0 & -h_{z} & -h_{y} \\
0 & J_{x}+J_{z} & 0 & -h_{z} & 0 & h_{x} \\
0 & 0 & J_{x}+J_{y} & -h_{y} & -h_{x} & 0 \\
0 & -h_{z} & -h_{y} & J_{y}+J_{z} & 0 & 0 \\
-h_{z} & 0 & -h_{x} & 0 & J_{x}+J_{z} & 0 \\
-h_{y} & h_{x} & 0 & 0 & 0 & J_{x}+J_{y}
\end{array}\right]\left[\begin{array}{c}
\cos \theta \cos \phi \\
\cos \theta \sin \phi \\
\sin \theta \cos \beta \\
\cos \alpha \sin \phi \\
\cos \alpha \cos \phi \\
\sin \alpha \sin \beta
\end{array}\right]=0 .
$$

In order to survey the diversity of solutions, it is sufficient to study the special cases with the field in the $x y$ plane, i.e. $\Theta=\pi / 2$, for (i) $J_{x} \geq J_{y} \geq J_{z}$, (ii) $J_{z} \geq J_{x} \geq J_{z}$, and (iii) $J_{y} \geq J_{z} \geq J_{x}$. The results for a definite ordering $J_{x} \geq J_{y} \geq J_{z}$ with the field in either of the three coordinate planes may then be obtained by a suitable transformation of axes.

In case (i) $\left(J_{x} \geq J_{y} \geq J_{z}\right)$ the Néel state configuration obviously lies in the $x y$ plane, i.e. $\theta=\pi / 2, \alpha=0$. The angle $\phi$ characterizes the direction of the net magnetization, and $2 \beta$ is the angle between the directions of the sublattice magnetizations. The solution of (2.16) yields

$$
\begin{aligned}
& \tan \phi=\tan \Phi \frac{J_{x}+J_{z}}{J_{y}+J_{z}}, \\
& \cos \beta=\left[\frac{\left(J_{x}+J_{z}\right)\left(J_{y}+J_{z}\right)\left[\left(J_{x}+J_{z}\right) \sin ^{2} \Phi+\left(J_{y}+J_{z}\right) \cos ^{2} \Phi\right]}{\left(J_{x}+J_{y}\right)\left[\left(J_{x}+J_{z}\right)^{2} \sin ^{2} \Phi+\left(J_{y}+J_{z}\right)^{2} \cos ^{2} \Phi\right]}\right]^{1 / 2} .
\end{aligned}
$$

In case (ii) $\left(J_{z} \geq J_{x} \geq J_{z}\right)$ the sublattice spins lie in a plane perpendicular to the xy plane. Here, we have $\theta=\pi / 2, \beta=0$, and the angle between the directions of the two sublattice magnetizations is $2 \alpha$. Eqs. (2.16) are then solved by (2.17) and

$$
\cos \alpha=\left[\frac{\left(J_{x}+J_{y}\right)\left[\left(J_{x}+J_{z}\right)^{2} \sin ^{2} \Phi+\left(J_{y}+J_{z}\right)^{2} \cos ^{2} \Phi\right]}{\left(J_{x}+J_{z}\right)\left(J_{y}+J_{z}\right)\left[\left(J_{x}+J_{z}\right) \sin ^{2} \Phi+\left(J_{y}+J_{z}\right) \cos ^{2} \Phi\right]}\right]^{1 / 2} .
$$

The case (iii) $\left(J_{y} \geq J_{z} \geq J_{x}\right)$ shows more complex behavior since we know from (i) and (ii) that for $\Phi=0$ the spins of the two sublattices lie in the $x y$-plane, whereas for $\Phi=\pi / 2$ they are pointing out of the $x y$-plane. In fact there is a critical field direction $\Phi_{F}$ given by

$$
\tan \Phi_{F}=\frac{J_{y}+J_{z}}{J_{x}+J_{z}}\left[\frac{J_{y}-J_{z}}{J_{z}-J_{x}}\right]^{1 / 2},
$$


where one type of behavior changes into the other. It is a continuous transition. For $\Phi<\Phi_{F}$ the spin configuration is characterized by (2.17) and (2.18) with spins in the $x y$ plane. At $\Phi=\Phi_{F}$ the angle $\beta$ vanishes and the ground state is ferromagnetic with spins pointing in direction $\phi_{F}$ determined by

$$
\tan \phi_{F}=\left[\frac{J_{y}-J_{z}}{J_{z}-J_{x}}\right]^{1 / 2}
$$

which, in general, is not the direction of the field. For $\Phi>\Phi_{F}$ the configuration is given by (2.17) and (2.19), i.e. lying in a plane perpendicular to the plane of the configuration at $\Phi<\Phi_{F}$. These results, transformed by suitable rotations of axes to fixed ordering $J_{x}>J_{y}>J_{z}$, are illustrated in Fig. 1. As a remarkable result we have thus found that in the general anisotropic quantum AFM (1.1) there is always a special field direction $\left[\Phi=0, \Theta=\Theta_{F}\right.$ for $\left.J_{x} \geq J_{y} \geq J_{z}\right]$ where the GS is ferromagnetic in a finite field.

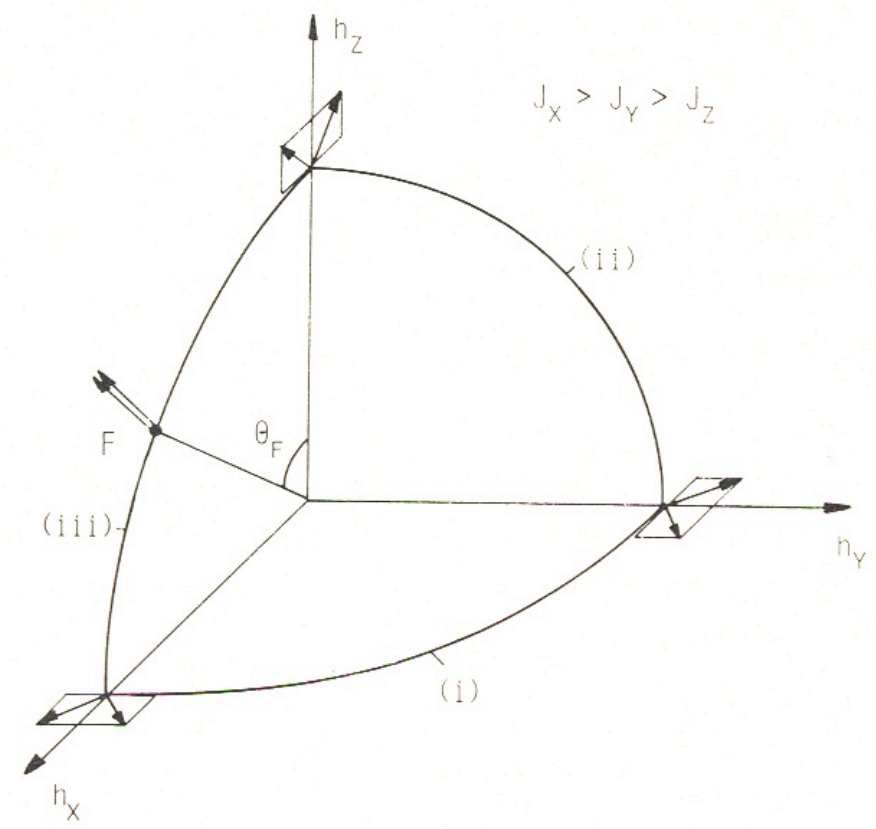

Figure 1. The figure shows one octant of the ellipsoidal surface (2.7) where the GS of Hamiltonian (1.1) is of the simple Néel-type. The configurations of the two sublattices are indicated by arrows for fields along the coordinate axis and for the special point F. (i), (ii) and (iii) refer to the cases discussed in detail in the text. Note that both inside and outside the ellipsoid the GS is, in general, not of the Néel-type but has a complicated structure due to fluctuations.

This general picture simplifies when $\mathcal{H}$ is chosen more symmetric. For the Heisenberg AFM $\left(J_{x}=J_{y}=J_{z}=J\right)$ the ellipsoid $(2.7)$ becomes a sphere with radius $h_{N}=2 J$ and the GS is ferromagnetic for all directions. For the isotropic $X Y$ model $\left(J_{x}=J_{y}=J, J_{z}=0\right)$ the ellipsoid is disk-like. The GS on the poles $\left(h=h_{z}\right)$ is ferromagnetic whereas on the equator the spins of both sublattices lie in the $x y$-plane at right angles to one another. For the Ising model $\left(J_{x}=J_{y}=\right.$ $\left.0, J_{z}=J\right)$ the ellipsoid degenerates into a line. The spins of the two sublattices are antiparallel for $\left|h_{N}^{z}\right|<J, h_{N}^{x}=h_{N}^{y}=0$ and become parallel for $h_{N}^{z}=J$.

The existence of a Néel GS in the quantum AFM is an extraordinary phenomenon. Generally, the GS is very complicated both inside and outside the ellipsoidal surface, and only at $\mathbf{h}=\mathbf{h}_{N}$ it behaves quasi-classically. The only exceptions are systems with $\left[\mathcal{H}_{\mathrm{EX}}, \mathcal{H}_{\mathrm{ZE}}\right]=0$, where the GS stays simply ferromagnetic for all $|\mathbf{h}|>\left|\mathbf{h}_{N}\right|$. The question arises, how these quasiclassical features of the quantum chain at $|\mathbf{h}|=\left|\mathbf{h}_{N}\right|$ are embedded in the behavior of the classical chain. In the 
following section we shall therefore briefly discuss the $T=0$ properties of the classical anisotropic AFM with special emphasis on the study of the role of $h_{N}$.

\section{Role of $h_{N}$ in the classical limit}

The GS properties of the classical anisotropic AFM (1.1) have thoroughly been studied in the past [15]. Here we summarize a few results which are important for our purpose. In the classical chain the spin operators $\mathbf{S}_{l}$ of (1.1) are replaced by vectors

$$
\mathbf{S}_{l}=s\left(\sin \theta_{l} \cos \phi_{l}, \sin \theta_{l} \sin \phi_{l}, \cos \theta_{l}\right) .
$$

The classical GS is found by minimizing $\mathcal{H}$ with respect to the polar angles $\left\{\theta_{l}, \phi_{l}\right\}$. A sketch of the phase diagram for the ordering $J_{x}>J_{y}>J_{z}$ is shown in Fig. 2. There is a closed surface $\mathbf{h}=\mathbf{h}_{s}$ separating the ferromagnetic (FM) phase outside from states with nonzero AFM LRO inside. In the $\left(h_{x}, h_{z}\right)$-plane there exists a line $\mathbf{h}=\mathbf{h}_{\mathrm{SF}}$ given by the hyperbola

$$
\frac{h_{x}^{2}}{J_{x}^{2}-J_{y}^{2}}-\frac{h_{z}^{2}}{J_{y}^{2}-J_{z}^{2}}=(2 s)^{2},
$$

where the sublattice magnetizations change their directions discontinuously in a spin-flop (SF) transition. The SF-phase boundary $\mathbf{h}=\mathbf{h}_{\mathrm{SF}}$ terminates in a bicritical point $\mathrm{B}$ on the FM phase boundary $\mathbf{h}=\mathbf{h}_{s}$.

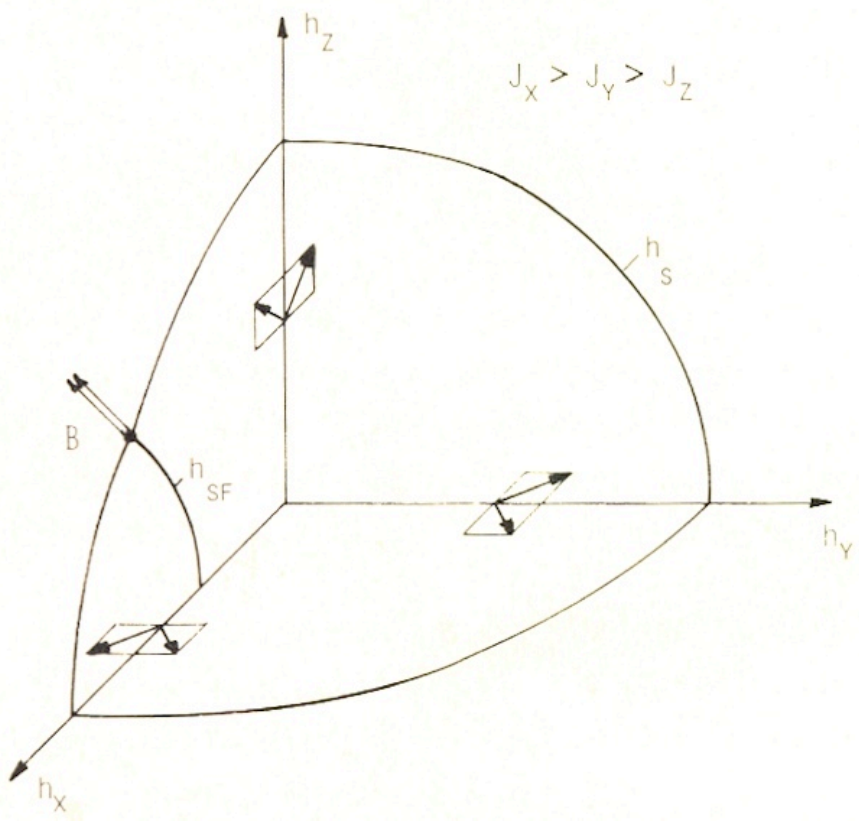

Figure 2. Zero-temperature phase diagram of the classical anisotropic AFM (1.1) in a magnetic field. The surface $\mathbf{h}=\mathbf{h}_{s}$, separates the ferromagnetic phase from two-sublattice Néel-type states inside. In the $\left(h_{x}, h_{z}\right)$-plane there exists a line $\mathbf{h}=\mathbf{h}_{\mathrm{SF}}$, where the sublattice magnetizations change their directions discontinuously in a spin-flop transition. The spin-flop phase boundary terminates in a bicritical point $\mathrm{B}$ on the ferromagnetic phase boundary. For fields along the coordinate axes and for the bicritical point $\mathrm{B}$, the configurations of the two sublattices are indicated by arrows.

The phase boundary $\mathbf{h}=\mathbf{h}_{s}$ for fields in the $x y$ plane is obtained as solution of the equation

$$
h_{x}^{2}+h_{y}^{2}=4 s^{2}\left(J_{x}+J_{y}\right)^{2}+\frac{4 s^{2}\left(J_{x}-J_{y}\right)^{2} h_{x}^{2} h_{y}^{2}}{4 h_{x}^{2} h_{y}^{2}+\left\{h_{x}^{2}-h_{y}^{2}-4 s^{2}\left(J_{x}^{2}-J_{y}^{2}\right)\right\}^{2}} .
$$


For weak anisotropy $s\left(J_{x}-J_{y}\right) \ll h_{s}$ this curve deviates only slightly from a circle:

$$
h_{x}^{2}=4 s^{2}\left(J_{x}+J_{y}\right)^{2}+s^{2}\left(J_{x}-J_{y}\right)^{2} \sin 2 \Phi, \quad \tan \Phi=h_{y} / h_{x} .
$$

For fields in the $y z$ plane, $\mathbf{h}_{s}$ describes an elliptic curve

$$
\frac{h_{x}^{2}}{4 s^{2}\left(J_{x}+J_{z}\right)^{2}}+\frac{h_{y}^{2}}{4 s^{2}\left(J_{y}+J_{z}\right)^{2}}=1
$$

For fields in the $x z$ plane, the saturation field lies on different curves on either side of the bicritical point. The part joining the $x$-axis has the structure of (3.4) and the part joining the $z$-axis the structure (3.2), both with indices changed accordingly. For $h$ parallel to any of the coordinate axes, the saturation field

$$
h_{s}^{x}=2 s\left\{J_{x}+\max \left(J_{y}, J_{z}\right)\right\}, \quad \text { etc, }
$$

is always larger than the Néel-state field

$$
h_{N}^{x}=2 s\left[\left(J_{x}+J_{y}\right)\left(J_{x}+J_{z}\right)\right]^{1 / 2}, \quad \text { etc, }
$$

except for the case of rotational symmetry around the field axis, for which $\left[\mathcal{H}_{\mathrm{EX}}, \mathcal{H}_{\mathrm{ZE}}\right]=0$. Here the two values coincide. Evidently the Néel-state ellipsoid $\mathbf{h}=\mathbf{h}_{N}$ is located in the interior of the surface $\mathbf{h}=\mathbf{h}_{s}$. For anisotropic cases the two surfaces have only the bicritical point B in common. In fact, the point $\mathrm{B}$ in the phase diagram of the classical chain (Fig. 2) coincides with the special point $\mathrm{F}$ on the surface $\mathbf{h}=\mathbf{h}_{N}$ (Fig. 1). We have verified that the classical GS configurations for fields $\mathbf{h}=\mathbf{h}_{N}$ are identical to the configurations found in section 2 by a quantum-mechanical calculation.

By looking only at the GS of the classical chain, the Néel field $\mathbf{h}_{N}$ is not distinguished in any way. The characteristic features showing up at $\mathbf{h}=\mathbf{h}_{N}$ in the quantum chain, appear, however, already when we introduce quantum fluctuations about the classical GS. Linear spin-wave theory yields the normal modes and the linear response function of the classical Hamiltonian (1.1). Quantum fluctuations are then obtained via the quantum-mechanical fluctuation-dissipation theorem. We shall discuss here the case $J_{x}>J_{y}>J_{z}, h=h_{z}$. The GS is of the SF type with sublattice spins in the xz plane as indicated in Fig. 2. For $h$ below the saturation field $h_{s}^{z}=2 s\left(J_{x}+J_{z}\right)$ the angle $2 \alpha$ between the spins of the two sublattices is given by $\cos \alpha=h / h_{s}^{z}$. Starting with this GS, linear spin-wave theory yields the following energies for the normal modes contributing, respectively, to the fluctuations parallel and perpendicular to the field direction:

$$
\begin{aligned}
& \omega_{\|}(q)=2 s\left[\left(J_{x}-J_{y} \cos q\right)\left(J_{x}+\left[J_{z}-(h / 2 s)^{2} /\left(J_{x}+J_{z}\right)\right] \cos q\right)\right]^{1 / 2}, \\
& \omega_{\perp}(q)=2 s\left[\left(J_{x}+J_{y} \cos q\right)\left(J_{x}-\left[J_{z}-(h / 2 s)^{2} /\left(J_{x}+J_{z}\right)\right] \cos q\right)\right]^{1 / 2} .
\end{aligned}
$$

We use here a representation in the extended Brillouin zone $I q l \ll \pi$ (see Ref. [16]). It has the advantage that the fluctuation function $\Phi_{\mu \mu}(q, \omega)$, the Fourier transform of $\left\langle\left(S_{l}^{\mu}(t)-\left\langle S_{l}^{\mu}\right\rangle\right)\left(S_{l^{\prime}}^{\mu}-\right.\right.$ $\left.\left.\left\langle S_{l^{\prime}}^{\mu}\right\rangle\right)\right\rangle$ can be written in the simple form

$$
\begin{aligned}
& \Phi_{x x}(q, \omega)=2 \pi I_{x x}(q) \delta\left[\omega-\omega_{\perp}(q)\right], \\
& \Phi_{y y}(q, \omega)=2 \pi I_{y y}(q) \delta\left[\omega-\omega_{\perp}(q)\right], \\
& \Phi_{z z}(q, \omega)=2 \pi I_{z z}(q) \delta\left[\omega-\omega_{\|}(q)\right],
\end{aligned}
$$

The fluctuation intensities are obtained by calculating also the linear-response function in the spin-wave approximation and applying the fluctuation-dissipation theorem. An equivalent but even simpler way $[16,17]$ is to use the fact that the first frequency moment of $\Phi_{\mu \mu}(q, \omega)$

$$
\left.K_{\mu \mu}(q)=\int_{0}^{\infty} \frac{d \omega}{2 \pi} \omega \Phi_{\mu \mu}(q, \omega)=-\frac{1}{2}\left\langle\left[\mathcal{H}, S^{\mu}(q)\right], S^{\mu}(-q)\right]\right\rangle
$$


is exactly known in terms of the magnetization $\sigma_{z}$ and the static nearestneighbour correlation functions $F_{\mu}=\left\langle S_{l}^{\mu} S_{l+1}^{\mu}\right\rangle$,

$$
\begin{aligned}
& K_{x x}(q)=-J_{y}\left(F_{y}-F_{z} \cos q\right)-J_{z}\left(F_{z}-F_{y} \cos q\right)+\frac{1}{2} h \sigma_{z}, \\
& K_{y y}(q)=-J_{x}\left(F_{x}-F_{z} \cos q\right)-J_{z}\left(F_{z}-F_{x} \cos q\right)+\frac{1}{2} h \sigma_{z}, \\
& K_{z z}(q)=-J_{x}\left(F_{x}-F_{y} \cos q\right)-J_{y}\left(F_{y}-F_{x} \cos q\right) .
\end{aligned}
$$

For the classical GS at $h \leq h_{s}^{z}$ we have $\sigma_{z}=s \cos \alpha, F_{x}=-s^{2} \sin ^{2} \alpha, F_{y}=0, F_{z}=s^{2} \cos ^{2} \alpha$, $\cos \alpha=h / h_{s}^{z}$. With these ingredients we obtain from (3.8), (3.9) and (3.10) the following fluctuation intensities:

$$
\begin{aligned}
& I_{x x}(q)=\frac{1}{2} s\left(\frac{h}{h_{s}^{z}}\right)^{2}\left[\frac{J_{x}+J_{y} \cos q}{J_{x}-\left[J_{z}-(h / 2 s)^{2} /\left(J_{x}+J_{z}\right)\right] \cos q}\right]^{1 / 2}, \\
& I_{y y}\left(q=\frac{1}{2} s\left[\frac{J_{x}-\left[J_{z}-(h / 2 s)^{2} /\left(J_{x}+J_{z}\right)\right] \cos q}{J_{x}+J_{y} \cos q}\right]^{1 / 2},\right. \\
& I_{z z}(q)=\frac{1}{2} s\left\{1-\left(h / h_{s}^{z}\right)^{2}\right\}\left[\frac{J_{x}-J_{y} \cos q}{J_{x}+\left[J_{z}-(h / 2 s)^{2} /\left(J_{x}+J_{z}\right)\right] \cos q}\right]^{1 / 2} .
\end{aligned}
$$

By studying the field dependence of these functions we observe the following:

(i) At $h=h_{N}^{z}$ all three components $I_{\mu \mu}(q)$ of the fluctuation intensities are constant, i.e. the fluctuations are completely uncorrelated. This means that the correlations in real space are $\delta$-functions without decaying terms despite the presence of fluctuations.

(ii) Generally, the spectrum (3.7) is gapless only at $h=h_{s}^{z}$. Here the transverse fluctuations $\left(I_{x x}\right)$ are divergent. This reflects the phase transition where AFM LRO is destroyed.

In conclusion we may say that the Néel-type GS's of the classical chain are altered by the introduction of quantum fluctuations except for field on the ellipsoid $\mathbf{h}=\mathbf{h}_{N}$.

\section{The critical field}

In this section, we study the critical surface $\mathbf{h}=\mathbf{h}_{c}$ which separates GS's with true or incipient AFM LRO from GS's without AFM LRO. In the classical limit $s \rightarrow \infty$, this transition occurs at the saturation field $\mathbf{h}_{s}$, and the results of section 3 show that the values of the Néel field are generally smaller than those of the critical field $h_{s}$ for the same direction. There are two exceptional cases where $\mathbf{h}_{N}=\mathbf{h}_{s}=\mathbf{h}_{c}$ (for arbitrary $s$ ): (i) in the bicritical point B; (ii) in the rotationally symmetric case $\left[\mathcal{H}_{E X}, \mathcal{H}_{Z E}\right]=0$. For the other cases the behavior becomes more complex for $s<\infty$. Here the quantum fluctuations are expected to have two major effects: (i) there is no longer a finite saturation field; (ii) the critical field $h_{c}$ is reduced from its classical value $h_{s}$.

In order to localize the true critical field in the quantum case, we use our result of section 2 that the AFM order parameter is non-zero at $\mathbf{h}=\mathbf{h}_{N}$. Thus, for a given field direction $h_{n}$ is a lower bound for the critical field. On the other side, it is highly plausible to use as upper bound the classical saturation field $h_{s}$, for it is a reasonable assumption that the quantum fluctuations will diminish the ordering and therefore reduce the value of $h_{c}$. Hence, we have

$$
h_{N} \leq h_{c} \leq h_{s} .
$$

This localization of $\mathbf{h}_{c}$ is perfect for isotropic systems (where $h_{N}=h_{s}$, and still fairly efficient for weak anisotropies as is implicit in the analytic expressions for $h_{N}$ and $h_{s}$. A case with relatively strong anisotropy $\left(J_{x}=1.5, J_{y}=1.0, J_{z}=0.5\right)$ is illustrated in fig. 3 . It shows the intersections of both surfaces $\mathbf{h}=\mathbf{h}_{N}$ and $\mathbf{h}=\mathbf{h}_{s}$ as sketched in figs. 1 and 2, respectively, with the three coordinate planes.

Apart from $\mathbf{h}_{N}, \mathbf{h}_{s}, \mathbf{h}_{c}$ there is a further characteristic field in the system described by the Hamiltonian (1.1). It is obtained from the energy gap $\Delta e$ between the GS and the lowest excited 


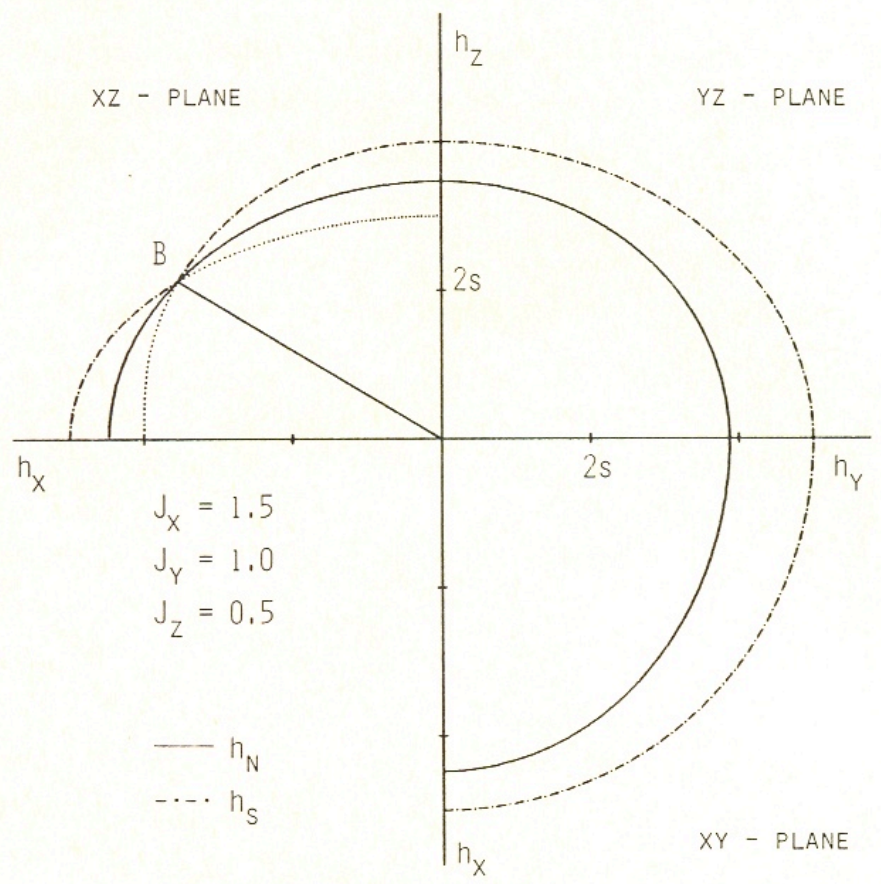

Figure 3. Intersections of the surfaces $\mathbf{h}=\mathbf{h}_{N}$ (Néel-state field) and $\mathbf{h}=\mathbf{h}_{s}$, (classical saturation field), respectively, with the three coordinate planes. The true critical field $\mathbf{h}=\mathbf{h}_{c}$ is expected to lie between the two surfaces; in particular it will contain the bicritical point $\mathrm{B}$.

state for $h \gg J_{\mu}$. A perturbation theory to first order in $J_{\mu} / h$ yields $\Delta E=h-h_{A}+\mathrm{O}\left(h^{-1}\right)$ which defines a field $h_{A}$ where the linear dependence extrapolates to zero. The explicit calculation yields for arbitrary $s$ (with $\Theta, \Phi$ the polar angles of the field)

$$
h_{A}=s\left(J_{x}+J_{y}+J_{z}\right)+s\left(J_{x} \sin ^{2} \Theta \cos ^{2} \Phi+J_{y} \sin ^{2} \Theta \sin ^{2} \Phi+J_{x} \cos ^{2} \Theta\right) .
$$

An expansion of (2.8) for weak anisotropies $\epsilon_{x}=J_{x} / J_{z}-1, \epsilon_{y}=J_{y} / J_{z}-1\left(J_{z} \equiv J\right)$ yields a positive definite quadratic form for $h_{A}-h_{N}$ :

$$
\begin{gathered}
h_{A}-h_{N}=\frac{1}{2} H_{x x} \epsilon_{x}^{2}+H_{x y} \epsilon_{x} \epsilon_{y}+\frac{1}{2} H_{y y} \epsilon_{y}^{2}, \\
H_{x x}=s J\left(1-\sin ^{2} \Theta \cos ^{2} \Phi\right)-\frac{3}{4} s J\left(1-\sin ^{2} \Theta \cos ^{2} \Phi\right)^{2}, \\
H_{y y}=s J\left(1-\sin ^{2} \Theta \sin ^{2} \Phi\right)-\frac{3}{4} s J\left(1-\sin ^{2} \Theta \sin ^{2} \Phi\right)^{2}, \\
H_{x y}=\frac{1}{2} s J\left(1-\sin ^{2} \Theta\right)-\frac{3}{4} s J\left(1-\sin ^{2} \Theta \cos ^{2} \Phi\right)\left(1-\sin ^{2} \Theta \cos ^{2} \Phi\right) .
\end{gathered}
$$

Hence, we have $H_{N} \leq h_{A}$, at least for weak anisotropies. In cases with the field parallel to any of the coordinate axes (e.g. $h=h_{z}$ ) we obtain for the three characteristic fields

$$
h_{N}^{z}=2 s \sqrt{\left(J_{x}+J_{z}\right)\left(J_{y}+J_{z}\right)}, \quad h_{A}^{z}=s\left(J_{x}+J_{y}+2 J_{z}\right), \quad H_{s}^{z}=2 s\left[J_{z}+\max \left(J_{x}, J_{y}\right)\right] .
$$

Obviously they satisfy the relation (for arbitrary anisotropy)

$$
h_{N}^{z} \leq h_{Z}^{z} \leq h_{s}^{z}
$$




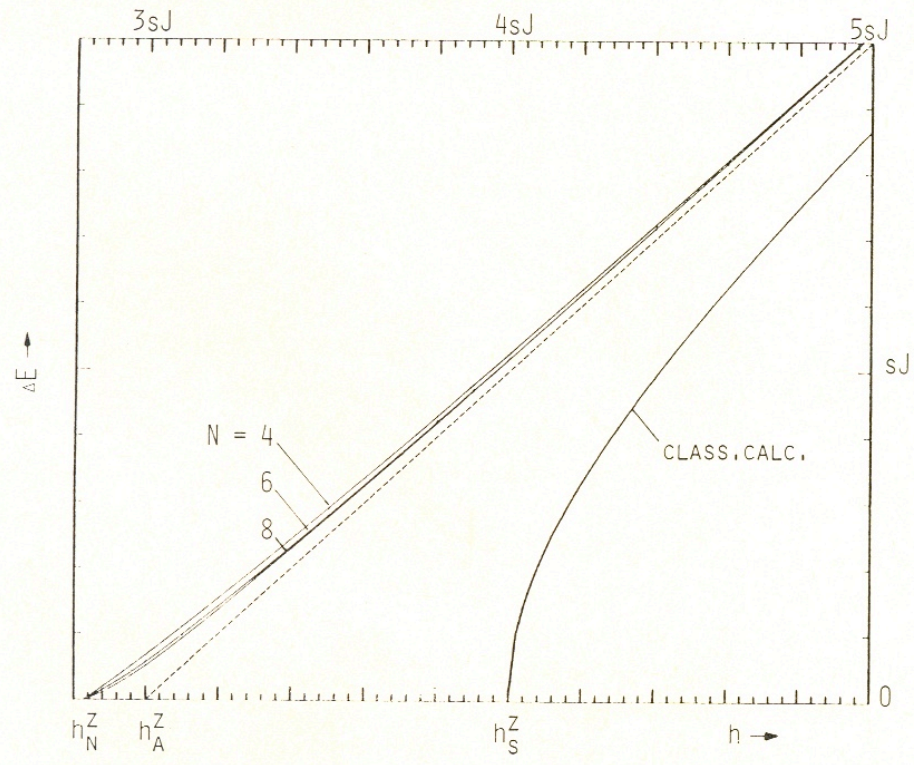

Figure 4. Energy gap $\Delta E$ between the singlet GS and the lowest excited state for the system (1.1) with $J_{x}=J_{z}=J, J_{y}=0, h>h_{N}^{z}$. The lowest full line is the result (4.7) for the classical chain. The other three full lines represent finite-chain results for $s=\frac{1}{2}$ systems of lengths $N=4,6,8$, respectively. For large fields all these lines approach the dashed line representing $\Delta E=h-h_{A}^{z}$ of (4.5).

The equality signs hold if $\left[\mathcal{H}_{E X}, \mathcal{H}_{Z E}\right]=0$, i.e. if $J_{x}=J_{y}$.

The possible significance of $h_{A}$ is connected with the fact that we expect the GS to be twofold degenerate (for $N \rightarrow \infty$ ) in the whole region $0 \leq h \leq h_{c}$ due to the presence of AFM LRO, and to become nondegenerate at $h>h_{c}$ when the AFM ordering is suppressed. We therefore study the field dependence of the energy gap $\Delta e$ between the GS and the lowest excitation for large $h$. For a representative example $\left(J_{x}=J, J_{y}=0, J_{z}=J, h=h_{z}\right)$ this is illustrated in fig. 4 . In the classical limit $s \rightarrow \infty, \Delta E(h)=\omega_{\|}(0)=\omega_{\perp}(\pi)$ can be calculated exactly in the framework of linear spinwave theory, yielding

$$
\Delta E(h)=\left[\left(h-h_{s}^{z}\right)\left(h-h_{s}^{z}+2 s\left|J_{x}-J_{y}\right|\right)\right]^{1 / 2}, \quad h=h_{z} .
$$

It vanishes at $h=h_{s}^{z}$, the critical field for $s \rightarrow \infty$, as shown in fig. 4. For large $h,(4.7)$ approaches the same asymptotic behavior $\Delta E(h) \sim h-h_{A}^{z}$, (dashed line in fig. 4) as obtained above for the quantum chain. The corresponding characteristic field $h_{A}^{z}$ lies, however, clearly below the true critical field $h_{s}^{z}$ of the classical chain. Therefore, $h_{A}^{z}$ has no obvious physical significance in the limit $s \rightarrow \infty$. In the quantum chain, $\Delta E(h))$ is not known except for its asymptotic behavior at $h \gg J_{\mu}$, as calculated above. For $s=\frac{1}{2}$, we can resort to finite-chain calculations on systems with up to $N=8$ spins for further information 1 Fig. 4 shows such finite-chain results for the above example. For finite $N$, the gap $\Delta E(h)$ is zero only at $h=h_{N}$ and at a discrete set of special fields below $h_{N}$. On the other hand, by use of our exact results for $h=h_{N}$, we can prove that the slope of $\Delta E(h)$ at $h=h_{N}$ goes to zero in the thermodynamic limit. This indicates that for $N \rightarrow \infty$ the GS may stay twofold degenerate in a finite interval $h_{N} \leq h \leq h_{c}$ beyond the Néel-state field $h_{N}$. The remarkable feature of the finite-chain results in fig. 4 is that the corresponding curves for the gap energy do not cross the dashed line $\Delta E(h)=h-h_{A}^{z}$. Although we cannot prove that for larger $N$ this crossing cannot occur, the rapid convergence for $h>h_{A}^{z}$ is indicative that it does not. Analogous calculations on a variety of systems with different anisotropies confirm this observation.

\footnotetext{
${ }^{1}$ Details about the method of finite-chain calculations are found in ref. 18 and in references therein.
} 
Therefore, it is highly suggestive to conjecture that for $s=\frac{1}{2}, \Delta E(h)=h-h_{A}^{z}$, is a lower bound for the true energy gap, or equivalently, that $h_{A}^{z}$ of (4.5) is an upper bound for the critical field.

As testing ground for this conjecture we can use the result of Barouch and McCoy [11] for the anisotropic XY model with $h=h_{z}$, the only nontrivial case where the critical field is exactly known:

$$
h_{c}^{z}=\frac{1}{2}\left(J_{x}+J_{y}\right) .
$$

It coincides with $h_{A}^{z}$, of (4.7) for $J_{z}=0$ and hence is consistent with our conjecture. For systems with $s>\frac{1}{2}$ the critical field $h_{c}^{z}$ is expected to be closer to the classical saturation field $h_{s}^{z}$. Thus $h_{A}^{z}$ will certainly lose its significance which it may have for $s=\frac{1}{2}$. It would be interesting to have finite-chain results for systems with $s>\frac{1}{2}$ in order to illustrate this point.

\section{Phase diagrams}

Here we use the results of the preceding sections for a discussion of the variety of features appearing in the phase diagrams of the $1 \mathrm{D}$ quantum AFM (1.1) at $T=0$ in a magnetic field. So far exact results have been available only for the anisotropic $s=\frac{1}{2} \mathrm{XY}$ model $\left(J_{z}=0\right)$ [10] and the uniaxial $s=\frac{1}{2} \mathrm{XXZ}$ model $\left(J_{x}=J_{y}\right)$ [8] both with field in the $z$-direction. The former system can be mapped onto a system of noninteracting fermions, the latter model is amenable to Bethe-Ansatz calculations.

Before we discuss the phase diagrams for a few explicit examples, it is useful to characterize the various phases which are realized by (1.1) for $s=\frac{1}{2}$ with hh parallel to a coordinate axis. We distinguish five different phases:

(i) Antiferromagnetic (AFM) phase: It is characterized by a non-zero staggered magnetization parallel to the applied field. This phase is realized by all easy-axis systems (biaxial or uniaxial) in a field parallel to the easy axis.

(ii) Spin-flop (SF) phase: Here the AFM order parameter is perpendicular to $\mathbf{h}$. This phase is realized by uniaxial (easy-axis or easy-phase) systems in a field perpendicular to the axis, as well as by biaxial systems with $\mathbf{h}$ along any axis.

(iii) Incommensurate (INC) phase: It is realized only by systems with $\left[\mathcal{H}_{E X}, \mathcal{H}_{Z E}\right]=0$, i.e. by uniaxial systems with $\mathbf{h}$ parallel to the axis and by the isotropic Heisenberg model. In this phase, the rotational symmetry of $\mathcal{H}$ has the effect of enhancing the fluctuations resulting in the absence of true LRO. Nevertheless, ordering is present as incipient LRO in the powerlaw decay of the correlation functions $\left\langle S_{l}^{\mu} S_{l+R}^{\mu}\right\rangle \sim \cos \left(q_{\mu} R\right) \cdot R^{-(d-2+\eta)}$. As a remarkable result it has been found that the leading term in the asymptotic behavior of $\left\langle S_{l}^{\mu} S_{l+R}^{\mu}\right\rangle$ with $\mathbf{h}=\mathbf{h}_{\mu}$. corresponds to a mode with a wave number $q_{\mu}$ which is incommensurate to the underlying lattice structurel $[12,13]$. This wave number is related to the magnetization $\sigma_{\mu}$ through $q_{\mu}=\pi\left(1-2 \sigma_{\mu}\right)$.

(iv) Paramagnetic (PM) phase: In this phase the AFM ordering is suppressed by the field, but due to the quantum fluctuations arising from the noncommutability of $\mathcal{H}_{E X}$ and $\mathcal{H}_{Z E}$ the magnetization is not saturated. The correlation functions decay exponentially. The PM phase occurs in all systems with $\left[\mathcal{H}_{E X}, \mathcal{H}_{Z E}\right] \neq 0$.

(v) Ferromagnetic (FM) phase: Here the magnetization is saturated, and the fluctuations are uncorrelated. The FM state is an eigenstate in all systems with $\left[\mathcal{H}_{E X}, \mathcal{H}_{Z E}\right]=0$.

In the limit $h=0$, the AFM and SF phases are indistinguishable. Both are characterized by a doublet GS (in the thermodynamic limit), separated by an energy gap from the lowest band of excited states. In the INC phase there is no energy gap, and the GS is a singlet. Note that at $h=0$ the INC phase is not really INCommensurate but still displays INCipient LRO. Both the PM and the FM phase are realized in strong fields. There is a gap of magnitude $\Delta E \simeq h-h_{c}$ between the singlet GS and the lowest excitation. 
As typical representatives of anisotropic AFM chains we discuss the phase diagrams of the uniaxial XXZ model (see fig. 5) with field (a) parallel, (b) perpendicular to the axis, and the anisotropic XY model (see fig. 6) with (a) in-plane, (b) out-of-plane field. In two of the four cases, exact results are available for $s=\frac{1}{2}$, which may serve as testing-ground for our approach.
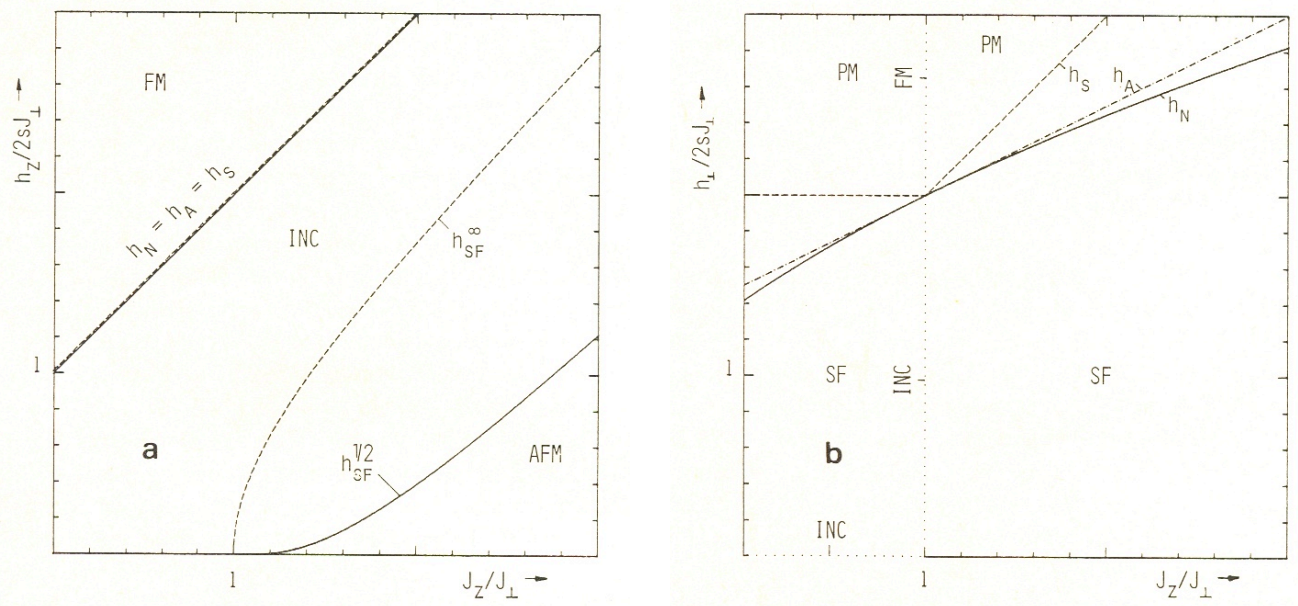

Figure 5. Zero-temperature phase diagram of the uniaxial XXZ model with field (a) parallel, (b) perpendicular to the axis. The various phases and critical fields are explained in the text.
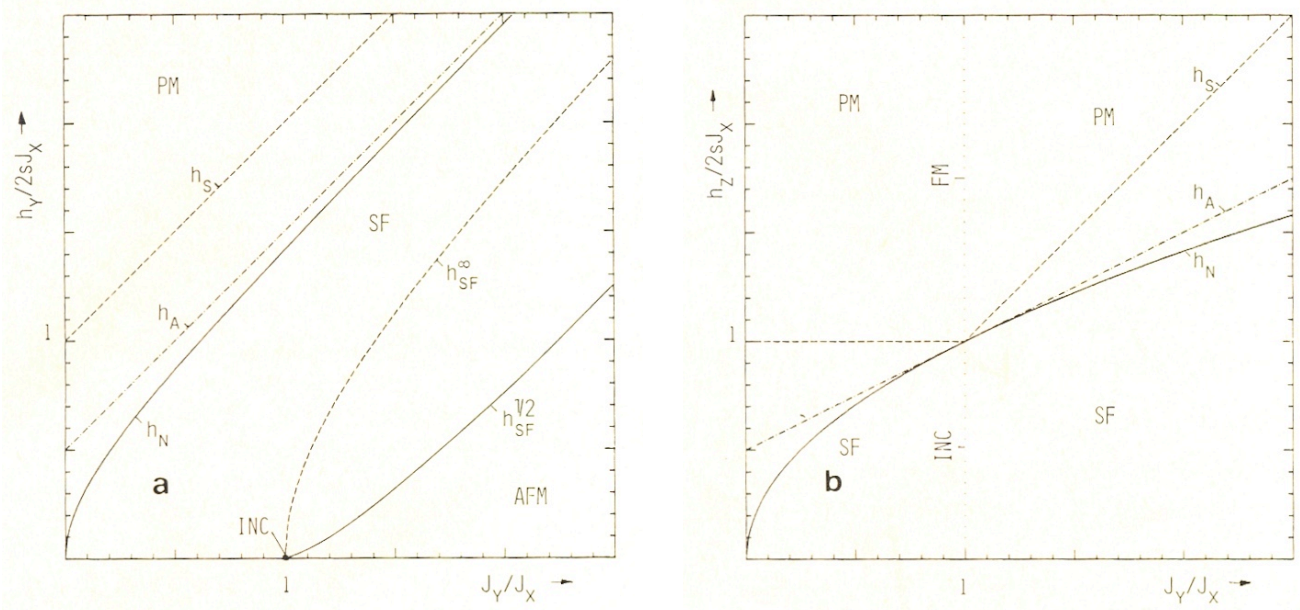

Figure 6. Zero-temperature phase diagram of the anisotropic XY model with (a) in-plane, (b) out-of-plane field. The various phases and critical fields are explained in the text.

5.1. $J_{x}=J_{y} \equiv J_{\perp}, h=h_{z}$ (fig. 5a)

Due to the rotational symmetry of $\mathcal{H}$, the critical field which separates the INC phase from the FM phase is exactly known,

$$
h_{c}=h_{N}=h_{A}=h_{s}=2 s\left(J_{\perp}+J_{z}\right) .
$$

For easy-plane anisotropy $\left(J_{z}<J_{\perp}\right)$ the INC phase stays stable down to $h=0$. For easy-axis anisotropy $\left(J_{z}>J \perp\right)$, the low-field phase is the AFM phase, separated from the INC phase by 
the discontinuous spin-flop transition at $h=h_{S F}^{s}$. The spin-flop field $h_{S F}^{s}$ strongly depends on the spin quantum number $s$. For $s=\frac{1}{2}, h_{S F}^{1 / 2}$ has been calculated exactly by Yang and Yang [8]. The classical result reads

$$
h_{S F}^{\infty}=2 s \sqrt{J_{z}^{2}-J_{\perp}^{2}} .
$$

We observe that the quantum fluctuations which are strongest for $s=\frac{1}{2}$ have the effect of destabilizing the AFM phase and shifting the SF transition to lower fields.

\section{2. $J_{x}=J_{y} \equiv J_{\perp}, h=h_{\perp}$ (fig. 5b)}

At $h=0$, there is incipient LRO for $J_{z} \leq J_{\perp}$, and true LRO for $J_{z}>J$. In the easy-plane case, an in-plane field induces a continuous transition from the INC phase to the SF phase, with an AFM order parameter going to zero for $h \rightarrow 0$. In the isotropic Heisenberg case $\left(J_{z}=J_{\perp}\right)$ the INC phase persists. Nevertheless the system behaves singularly at $h_{\perp}=0^{+}$which is due to the reduction of rotational invariance of $\mathcal{H}[6,8,13]$. The AFM ordering present in low fields vanishes at a critical field $h_{c}$ located between $h_{N}$ and $h_{s}$ :

$$
h_{N}=2 s \sqrt{2 J_{\perp}\left(J_{\perp}+J_{z}\right)}, \quad h_{s}=2 s\left[J_{\perp}+\max \left(J_{z}, J_{\perp}\right)\right] .
$$

In the classical limit the transition occurs at $h_{s}$, whereas for $s=\frac{1}{2}$ the critical field is expected according to our arguments of section 4 - to be located rather at or below

$$
h_{A}=s\left(3 J+J_{z}\right) .
$$

Coincidence of the critical field with $h_{A}$ for $s=\frac{1}{2}$ is confirmed by exact results only for $J_{z} / J_{\perp}=1$ and $J_{z} / J_{\perp}=\infty$.

\section{3. $J_{z}=0, h=h_{y}$ (fig. 6a)}

Here the low-field phase has SF character for $J_{y} / J_{x}<1$ and AFM character for $J_{y} / J_{x}>1$. As a special case the $h=0$ phase of the isotropic XY model $\left(J_{x}=J_{y}\right)$ has INC character. The AFM phase is destabilized at the spin-flop field $h_{S F}^{s}$, which again appears to be strongly $s$-dependent. In the classical limit it is given by

$$
h_{S F}^{\infty}=2 s \sqrt{\left|J_{x}^{2}-J_{y}^{2}\right|} .
$$

For $s=\frac{1}{2}$ it has been obtained numerically from extrapolations of finite-chain results. Again we observe that the AFM phase is destabilized at lower fields in the $s=\frac{1}{2}$ case, owing to quantum fluctuations. At higher fields the AFM LRO in the SF phase vanishes at the critical field $h_{c}$ located between

$$
h_{N}=2 s \sqrt{J_{x}\left(J_{x}+J_{y}\right)} \text { and } h_{s}=2 s\left(J_{x}+J_{y}\right) .
$$

We know that in the classical limit $h_{c}=h_{s}$, and for $s=\frac{1}{2}$ we expect

$$
h_{A}=s\left(J_{x}+2 J_{y}\right)
$$

to be an upper boundary for $h_{c}$.

\section{4. $J_{z}=0, h=h_{z}$ (fig. 6b)}

At $h=0$ there is true AFM LRO for $J_{y} / J_{x} \neq 1$ and incipient LRO in the isotropic case $J_{y} / J_{x}=1$. This ordering persists up to the critical field $h_{c}$ located between $h_{N}$ and $h_{s}$,

$$
h_{N}=2 s \sqrt{J_{x} J_{y}}, \quad h_{s}=2 s \max \left(J_{x}, J_{y}\right) .
$$


Here we know the exact critical field $h_{c}$ both in the classical and quantum limits [11]: For $s=\infty$, $h_{c}=h_{s}$, and for $s=\frac{1}{2}$

$$
h_{c}=h_{A}=\frac{1}{2}\left(J_{x}+J_{y}\right) .
$$

A general feature of the four examples discussed in this section is that in the classical system the low-field phases are stable up to higher fields than in 1 the $s=\frac{1}{2}$ system. This is in agreement with our argument used in section 4 that quantum fluctuations generally lower the value of the critical field.

\section{Conclusion}

We have investigated the GS properties of the anisotropic 1D spin-s AFM in a magnetic field of arbitrary direction. As an extraordinary phenomenon we have found that for a given field direction there always exists a particular field value $h_{N}$, where the GS behaves quasi-classically. For a given set of exchange parameters $J_{x}, J_{y}, J_{z}$, these special values $h_{N}$ lie on an ellipsoidal surface in field space. For fields both inside and outside this ellipsoid, the GS is, generally, very complicated due to the presence of quantum fluctuations. We have reviewed the phase diagram of the classical chain and shown that the special features occurring at $\mathbf{h}=\mathbf{h}_{N}$ are to some degree also present in the linear spin-wave approximation appropriate for $s \rightarrow \infty$. It was found that the ellipsoid $\mathbf{h}_{N}$ lies within the closed surface $\mathbf{h}_{s}$ representing the classical saturation field. We have demonstrated that $h_{N}$ and $h_{s}$ can be used as lower and upper bound, respectively, for the critical field $h_{c}$, where the (true or incipient) AFM LRO vanishes. Finally, we have characterized the variety of phases occurring in the anisotropic quantum spin chain at $T=0$, and discussed the phase diagrams for a few representative systems.

\section{Acknowledgments}

We are indebted to H. Beck, J.C. Bonner, H.W. Capel, D.C. Mattis, W. Pesch, M.W. Puga, H. Rohrer and R. Schilling for many valuable discussions. This work has been supported by the Swiss National Science Foundation. We have used a modified cmpj.sty style file.

\section{References}

1. For recent reviews with references to much of the relevant earlier literature see: J.C. Bonner, H.W.J. Blöte, H. Beck and G. Müller, Physics in One Dimension, J. Bernasconi and T. Schneider, eds. (Springer Series in Solid State Sciences Vol. 23 1981) p. 115. H. Beck, M.W. Puga and G. Müller, J. Appl. Phys. 52 (1981) 1998.

2. R.J. Baxter, Ann. Phys. (N.Y.) 70 (1972) 323.

3. J.D. Johnson, S. Krinsky and B.M. McCoy, Phys. Rev. A 8 (1973) 2526.

4. H.A. Bethe, Z. Phys. 71 (1931) 205.

5. L. Hulthén, Arkiv Mat. Astron. Fysik 26A (1938) 1.

6. R.B. Grifliths, Phys. Rev. 133 (1964) A768.

7. J. Des Cloizeaux and M. Gaudin, J. Math. Phys. 7 0966) 1384.

8. C.N. Yang and C.P. Yang, Phys. Rev. 150 (1966) 321,327; 151 (1966) 258.

9. E. Lieb, T. Schultz and D. Mattis, Ann. Phys. (N.Y.) 16 (1961) 406.

10. S. Katsura, Phys. Rev. 127 (1962) 1508.

11. E. Barouch and B.M. McCoy, Phys. Rev. A 3 (1971) 786.

12. N. Ishimura nd H. Shiba, Prog. Theor. Phys. 57 (1977) 1862.

13. G. Müller, H. Thomas, H. Beck and J.C. Bonner, Phys. Rev. B 24 (1981) 1429.

14. J. Kurmann, G. Müller, H. Thomas, M.W. Puga and H. Beck, J. Appl. Phys. 52 (1981) 1968.

15. C.J. Gorter and J. Haantjes, Physica 18 (1952) 285. J. Ubbink, Physica 19 (1953) 9. H. Rohrer and H. Thomas, J. Appl. Phys. 41 (1969) 1025. H. Thomas, Local properties at Phase Transitions, K.A. Müller and A. Rigamonti, eds. (Bologna, 1975) p. 659. For a recent systematic treatment of bilinear spin-wave theory see: C.A.M. Mulder, H.W. Capel and J.H.H. Perk, Physica 112B (1982) 147.

16. G. Müller, H. Thomas, M.W. Puga and H. Beck, J. Phys. C 14 (1981) 3399. 
17. P.C. Hohenberg and W.F. Brinkman, Phys. Rev. B 10 (1974) 128.

18. G. Müller and H. Beck, J. Phys. C ll (1978) 483. 